Check for updates

Cite this: Phys. Chem. Chem. Phys., 2020, 22, 22997

Received 9th August 2020, Accepted 25th September 2020

DOI: 10.1039/d0cp04205f

rsc.li/pccp

\title{
Quantifying how step-wise fluorination tunes local solute hydrophobicity, hydration shell thermodynamics and the quantum mechanical contributions of solute-water interactions $\uparrow$
}

\author{
João R. Robalo, (D) $\ddagger^{\mathrm{a}}$ Denilson Mendes de Oliveira, (D) $\ddagger^{\mathrm{b}}$ Petra Imhof, (D) $\S^{\mathrm{c}}$ \\ Dor Ben-Amotz (1D ${ }^{b}$ and Ana Vila Verde (D) $q^{\star a}$
}

\begin{abstract}
The ability to locally tune solute-water interactions and thus control the hydrophilic/hydrophobic character of a solute is key to control molecular self-assembly and to develop new drugs and biocatalysts; it has been a holy grail in synthetic chemistry and biology. To date, the connection between (i) the hydrophobicity of a functional group; (ii) the local structure and thermodynamics of its hydration shell; and (iii) the relative influence of van der Waals (dispersion) and electrostatic interactions on hydration remains unclear. We investigate this connection using spectroscopic, classical simulation and ab initio methods by following the transition from hydrophile to hydrophobe induced by the step-wise fluorination of methyl groups. Along the transition, we find that water-solute hydrogen bonds are progressively transformed into dangling hydroxy groups. Each structure has a distinct thermodynamic, spectroscopic and quantum-mechanical signature connected to the associated local solute hydrophobicity and correlating with the relative contribution of electrostatics and dispersion to the solute-water interactions.
\end{abstract}

\section{Introduction}

Cellular machinery, structural scaffolds, and compartments are essentially composed of sugars, amino acids, nucleobases and lipids whose functional groups, to put it simply, either form hydrogen bonds with each other and with water, or segregate from water. The balance of formed, perturbed and broken hydrogen bonds in the hydration shell - the balance of hydrophilic and

\footnotetext{
${ }^{a}$ Department of Theory \& Bio-Systems, Max Planck Institute for Colloids and Interfaces, Science Park, Potsdam 14476, Germany.

E-mail: ana.vilaverde@mpikg.mpg.de

${ }^{b}$ Purdue University, Department of Chemistry, West Lafayette, IN 47907, USA

${ }^{c}$ Institute for Theoretical Physics, Free University of Berlin, Arnimallee 14,

14195 Berlin, Germany

$\dagger$ Electronic supplementary information (ESI) available: Temperature dependence of the dangling $\mathrm{OH}$ peaks obtained after subtracting the background using a Gaussian fit; experimental Henry's law coefficients and hydration free energies for EtOH, MFE, DFE, and TFE; simulation hydration free energies for MFE, DFE, TFE; partial charges of the alcohol oxygen and the fluorines in MFE, DFE, TFE; probabilities of having exactly 1 or 2 hydrogen bond-like structures in the hydration shell of MFE, DFE, TFE; SAPT for water-bonded and hydrogen bondlike structures; tables with all simulation data presented in graphical form in the main text. See DOI: 10.1039/d0cp04205f

\$ These authors have contributed equally.

$\S$ Present address: Computer Chemistry Center, Friedrich-Alexander Universität (FAU) Erlangen-Nürnberg, Nägelsbachstraße 25, 91052 Erlangen, Germany.

T Present address: University of Duisburg-Essen, Faculty of Physics, Lotharstrasse 1, 47057 Duisburg, Germany.
}

hydrophobic interactions - influences self assembly, structure and functionality: ${ }^{1-7}$ life itself.

Tuning hydrophobicity by manipulating hydrogen bonds has been a longstanding goal and challenge in materials science, ${ }^{8-13}$ organic chemistry ${ }^{14-16}$ and supramolecular chemistry. ${ }^{17-20} \mathrm{How}^{-}$ ever, the usual strategies employed to modify hydrogen bond patterns (the simplest being methylation and hydroxylation) also result in changes in molecular surface area and topology, meaning that water-solute and water-water hydrogen bonds are simultaneously affected. The challenge in such strategies is often perceived as the prediction of which hydrogen bonds are broken, formed or perturbed, and how they will change a solute's hydrophobicity. We propose that this formulation is insufficient in that, at its essence, the challenge is also conceptual-to determine what is a hydrogen bond, what does it mean to perturb one in the context of a hydration shell, and what is its connection with local hydrophobicity. A hydration shell contains a distribution of hydrogen bond energies arising from mutual interactions of many water molecules and the solute that are, in turn, influenced by interactions with even more molecules. Importantly, the entropic contribution to hydrogen bond stability-a key factor in the theory of the hydrophobic effect-cannot be recovered from gas phase measurements. Here we address the above questions using direct measurements of hydration shell spectra, and calculations to enable the discretization of hydration shell structures, of systems with a set of chemical 
modifications resulting in stepwise changes in hydrophobicityenter fluorine.

The consecutive fluorination of a methyl group yields both a hydrophile (monofluoromethyl) and a hydrophobe (trifluoromethyl) only two H-to-F substitutions apart. ${ }^{21}$ The associated minimal changes to molecular surface area and the retention of molecular topology result in a minimal volume-induced structural perturbation of the hydration water molecules. ${ }^{21}$ Furthermore, classical calculations suggest that changes in the hydrophobicity of fluoromethyl groups along this series are mainly enthalpic. ${ }^{21}$ Interestingly, while mostly absent from biological systems, fluorine's forays into synthetic biology result in a remarkable ability to manipulate cellular machinery, ${ }^{22-25}$ structural motifs $^{26-28}$ and compartments. ${ }^{29,30}$ In chemistry, fluorination is routinely used as a provider of curious and useful characteristics, from the ability to form hydrogen bond donor carbon atoms ${ }^{31-34}$ to the unusual properties of perfluorinated oils. ${ }^{35-37}$

We combined experimental Raman multivariate curve resolution (Raman-MCR) spectroscopy, simulations using both classical force fields and density functional theory potentials, and quantummechanical calculations to quantify the changes in the hydrophobicity of a series of fluorinated solutes, the thermodynamics of putative hydrogen bonds in their hydration shells and the first principle contributions to water-solute interactions and hydration thermodynamics. Our chosen series of solutes is the simplest, most gradual, experimentally-accessible hydrophilicto-hydrophobic transition: 2-mono-, 2,2-di-, and 2,2,2-trifluoroethanol (MFE, DFE and TFE, respectively). We find that the hydration shell of each fluoromethyl group contains structures consisting of water hydroxy groups that point to the solute. We term these structures "hydrogen bond-like" because they obey typical geometric criteria used to identify hydrogen bonds albeit with distinct spectroscopic signatures and thermodynamic consequences. Along with the fact that the fluoromethyl groups range from hydrophilic to hydrophobic, the thermodynamic signature of these hydrogen bond-like structures leads to a distinction between enthalpically stabilized, de facto hydrogen bonds, entropically stabilized (for this reason termed "dangling") hydroxy groups, and hybrid structures. Likewise, the quantum mechanical nature of the solute-water attractive interactions also varies along the hydrogen bond-to-dangling hydroxy transition, further supporting a fundamental distinction between structurally similar hydration shell features.

\section{Methods}

\section{Experimental data collection and analysis methods}

Aqueous solutions of EtOH (200 proof, anhydrous, Decon Laboratories), MFE ( $\geq 95 \%$, Alfa Aesar), DFE ( $>98 \%$, Tokyo Chemical Industry), and TFE (99.8\%, Acros Organics) were prepared using ultrapurified water (Milli-Q UF plus, Millipore Inc., resistivity of $18.2 \mathrm{M} \Omega \mathrm{cm}$ ). In view of toxicity, all alcohol solutions were prepared in a fume hood and enclosed in sealed glass vials for Raman acquisition. Raman spectral measurements were performed as previously described ${ }^{38,39}$ at temperatures between $20{ }^{\circ} \mathrm{C}$ and $80{ }^{\circ} \mathrm{C}$ (held constant to within less than $\pm 0.1^{\circ} \mathrm{C}$ ) using an Ar-ion $514.5 \mathrm{~nm}$ excitation laser with $\sim 20 \mathrm{~mW}$ of power at the sample and $5 \mathrm{~min}$ of integration time.

Self-modeling curve resolution (SMCR) ${ }^{38,40,41}$ was used to obtain minimum area non-negative solute-correlated (SC) spectra from pairs of pure water and solution spectra that were collected under identical experimental conditions (as described above). Any residual baseline in the SC spectra was removed using either a linear or quadratic polynomial fit to selected background points adjacent to the Raman bands of interest. The dangling $\mathrm{OH}$ peaks, corresponding to water $\mathrm{OH}$ groups that do not participate in a normal water-water hydrogen bond, were identified in the SC spectra and quantified as previously described. ${ }^{42,43}$ Specifically, if the Raman scattering cross section of a dangling $\mathrm{OH}$ is assumed to be equal to the average Raman cross section of an $\mathrm{OH}$ group in pure water, we can obtain the average number $\langle k\rangle$ of those species using the following expression:

$$
\langle k\rangle=\left(\frac{2[\mathrm{~W}]}{[\mathrm{S}]}\right)\left(\frac{I_{\mathrm{D}-\mathrm{OH}}}{I_{\mathrm{W}-\mathrm{OH}}}\right)
$$

where $I_{\mathrm{D}-\mathrm{OH}}$ corresponds to the integrated area (measured counts) of a water dangling $\mathrm{OH}$ band, $I_{\mathrm{W}-\mathrm{OH}}$ is the integrated area of the $\mathrm{OH}$ band of pure water, $2[\mathrm{~W}]\left(\approx 2 \times 55.5 \mathrm{~mol} \mathrm{dm}^{-3}=\right.$ $\left.111 \mathrm{~mol} \mathrm{dm}^{-3}\right)$ is the concentration of $\mathrm{OH}$ groups in pure water, and $[\mathrm{S}]$ is the solute concentration in $\mathrm{mol} \mathrm{dm}^{-3}$ units.

A more general procedure for obtaining $\langle k\rangle$ relies on dividing the SC spectrum by the area of a solute intramolecular vibrational band, such as the $\mathrm{CH}$ stretch. In such a normalized spectrum, the dangling $\mathrm{OH}$ intensity is given by $I_{\mathrm{D}-\mathrm{OH}}^{\mathrm{CH}-n}=I_{\mathrm{D}-\mathrm{OH}} / I_{\mathrm{CH}}$. This last quantity can be directly translated into $\langle k\rangle$ given the values of $\Omega_{\mathrm{CH}}, \Omega_{\mathrm{OH}}$ and $n_{\mathrm{CH}}$, which are, respectively, the Raman cross sections of a single $\mathrm{CH}$ group and a single water $\mathrm{OH}$ group, and the number of $\mathrm{CH}$ bonds in a solute molecule, as follows:

$$
\langle k\rangle=n_{\mathrm{CH}}\left(\frac{\Omega_{\mathrm{CH}}}{\Omega_{\mathrm{OH}}}\right) I_{\mathrm{D}-\mathrm{OH}}^{\mathrm{CH}-n}
$$

The ratio $\Omega_{\mathrm{CH}} / \Omega_{\mathrm{OH}}$ in eqn (2) is estimated using the measured area of the $\mathrm{OH}$ band in pure water $\left(I_{\mathrm{W}-\mathrm{OH}}\right)$, the measured area of the $\mathrm{CH}$ band $\left(I_{\mathrm{CH}}\right)$ in the solute, and the concentrations of pure water and solute, as shown below:

$$
\frac{\Omega_{\mathrm{CH}}}{\Omega_{\mathrm{OH}}}=\left(\frac{I_{\mathrm{CH}}}{I_{\mathrm{W}-\mathrm{OH}}}\right)\left(\frac{2[\mathrm{~W}]}{n_{\mathrm{CH}}[\mathrm{S}]}\right)
$$

Note that eqn (2) is preferred over eqn (1) because normalization of the SC spectrum to the solute $\mathrm{CH}$ band area compensates for any variations in laser intensity (or drifts in optical alignment) that may occur during the Raman spectral acquisition process. Moreover, eqn (2) may also be used to correct the value of $\langle k\rangle$ for any difference between the Raman cross section of the dangling $\mathrm{OH}$ group and an average $\mathrm{OH}$ group in pure water. For example, previously reported calculations have predicted that some dangling $\mathrm{OH}$ groups may have a Raman cross section that is approximately half that of an average $\mathrm{OH}$ group in pure water. ${ }^{43}$ If that is assumed to also be the case for the dangling $\mathrm{OH}$ groups interacting with a solute fluorine atom, then the appropriate value of $\Omega_{\mathrm{CH}} / \Omega_{\mathrm{OH}}$ should be increased 
by a factor of two (relative to the value obtained using eqn (3)) and thus the resulting values of $\langle k\rangle$ (obtained using eqn (2)) would also increase by about a factor of two.

To obtain the dangling $\mathrm{OH}$ formation thermodynamics, we consider the process of forming one such dangling $\mathrm{OH}$ defect structure in a hydration shell that initially contained no dangling $\mathrm{OH}$ defects. This transformation was previously described using a lattice model ${ }^{43}$ which predicts that, as long as the dangling $\mathrm{OH}$ defect formation has a relatively low probability of occurrence (such that $\langle k\rangle<1$ ), the equilibrium constant $K$ for the dangling $\mathrm{OH}$ formation process should be well approximated by $\langle k\rangle$. Thus, the following expressions may be used to obtain the Gibbs energy, enthalpy, and entropy changes associated with the formation of dangling $\mathrm{OH}$ defects in the hydration-shell of the solute (where $R$ is the gas constant and $T$ is the absolute temperature):

$$
\begin{aligned}
\Delta G & =-R T \ln \langle k\rangle \\
\Delta H & =\left[\frac{\partial(\Delta G / T)}{\partial(1 / T)}\right]_{P} \\
\Delta S & =-\left(\frac{\partial \Delta G}{\partial T}\right)_{P}
\end{aligned}
$$

\section{Computational details}

\section{Non-water-bonded hydroxy structures in the hydration shell}

The present work is focused on the occurrence of two structures in the hydration shell of MFE, DFE or TFE: hydrogen bond-like hydroxy structures and 'free' hydroxy structures, shown in cartoon form in Fig. 1. A hydrogen bond-like structure is defined as a water hydroxy group that directs its hydrogen atom towards a fluorine or hydrogen atom in a $-\mathrm{CFH}_{2}$ (in MFE), $-\mathrm{CF}_{2} \mathrm{H}$ (DFE) or $-\mathrm{CF}_{3}$ (TFE) group, such that the distance, $d_{\mathrm{OW}-\mathrm{H} / \mathrm{F}}$, between the water oxygen atom and the alcohol hydrogen or fluorine atom is below $3.5 \AA$ and the angle $\theta$ between water oxygen, water hydrogen and alcohol fluorine/ hydrogen atoms falls within $160^{\circ} \leq \theta \leq 180^{\circ}$. $\|$ A free hydroxy group belongs to a water molecule whose oxygen atom lies no further than $5.69 \AA$ from the carbon atom in the fluoromethyl group (the first hydration shell radius of tetrafluoromethane ${ }^{21}$ ) and (i) does not form a hydrogen bond-like structure (see definition above); (ii) does not donate a hydrogen bond (present if $d_{\mathrm{OW}-\mathrm{H}}<3.5 \AA, 160^{\circ} \leq \theta \leq 180^{\circ}$ ) to the alcohol group of the solute; (iii) does not donate a hydrogen bond (present if $d_{\mathrm{OW}-\mathrm{H}}<3.5 \AA, 145^{\circ} \leq \theta \leq 180^{\circ}$ ) to any other water molecule in the simulation box. The collection of hydrogen bond-like structures and free hydroxys will here be referred to as non-water-bonded hydroxy structures. We refer the reader to ref. 42 for a comparison of different definitions employed in quantifying such structures. Free energies of formation of either structure can be calculated via $\langle k\rangle$ in eqn (4). In the case

\| In our prior publication concerning TFE and ethanol, ${ }^{42}$ this structure was termed "dangling"; the new nomenclature was adopted here because it is both more general and more descriptive, as well as easy to understand.

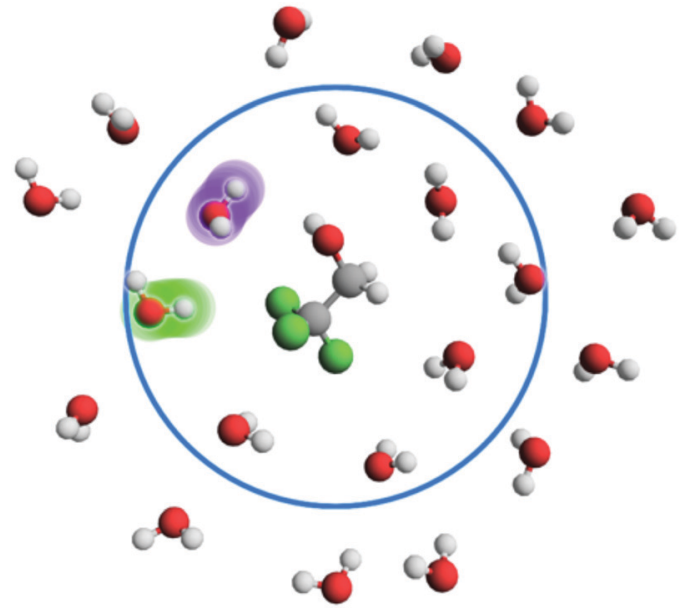

Fig. 1 Schematic representation of the hydration shell of TFE. A hydroxy group forming a hydrogen bond-like structure is highlighted in green, a free hydroxy group is highlighted in purple; we refer to both jointly as nonwater-bonded hydroxy groups. Carbon atoms are shown in gray, fluorine in green, oxygen in red and hydrogen in white. The solute and the first hydration shell waters are encircled in blue.

of non-water-bonded hydroxys, $\langle k\rangle$ equals the sum of hydrogen bond-like structures and excess free hydroxys in solution:

$$
\langle k\rangle=\mathrm{OH}_{\mathrm{NWB}}=\mathrm{OH}_{\mathrm{HBL}}+\Delta \mathrm{OH}_{\mathrm{Free}},
$$

where $\mathrm{OH}_{\mathrm{NWB}}$ stands for the average number of non-waterbonded hydroxy groups per hydration shell and $\mathrm{OH}_{\mathrm{HBL}}$ stands for the average number of hydrogen bond-like structures per hydration shell. $\Delta \mathrm{OH}_{\text {Free }}$ is the difference, at a given temperature, between the average number of free hydroxy groups in the fluoromethyl group's hydration shell $\left(\mathrm{OH}_{\mathrm{Free}}\right.$,solution $)$ and the average number of non-hydrogen bonded hydroxy groups that exist in pure water for as many water molecules as there are in the hydration shell of the fluoromethyl group $\left(\mathrm{OH}_{\text {Unbound,water }}\right)$ :

$$
\Delta \mathrm{OH}_{\text {Free }}=\mathrm{OH}_{\text {Free,solution }}-\mathrm{OH}_{\text {Unbound,water }}
$$

We have previously argued that taking $\langle k\rangle=\mathrm{OH}_{\mathrm{NWB}}$ is in best agreement with Raman-MCR measurements of solutions of 2,2,2-trifluoroethanol, ${ }^{42}$ implying that non-water-bonded hydroxys are representative of the population of spectroscopically active structures (near $3600 \mathrm{~cm}^{-1}$ ) in these systems. Eqn (4) was further used to calculate the free energy of formation of hydrogen bondlike structures, with $\langle k\rangle=\mathrm{OH}_{\mathrm{HBL}}$, which concern non-waterbonded hydroxy groups undergoing direct interactions with the fluoromethyl group.

\section{Molecular dynamics with classical force fields}

Each system consists of a cubic box of edge length $\approx 4 \mathrm{~nm}$ containing a single alcohol molecule-MFE, DFE or TFE (all data presented for TFE are taken from ref. 42)-solvated by water. Water is described with the TIP4P-Ew force field. ${ }^{44}$ The alcohols are modelled with a modified $\mathrm{GAFF}^{45}$ force field, where the Lennard-Jones parameters for the fluorine atoms are taken from ref. 46 , those of the hydrogen atoms in $-\mathrm{CFH}_{2}$ and $-\mathrm{CF}_{2} \mathrm{H}$ groups are taken from ref. 21 and those of the 
hydrogen atoms in $-\mathrm{CH}_{2}-$ groups were described in ref. 42 . All of these modified parameters were optimized against the hydration free energies of alkanes and fluoroalkanes. Initial atomic charges on MFE and DFE are calculated through a sequential MP2/6-31G*, RHF/6-31G* $\mathrm{RESP}^{47}$-fitting procedure, as employed for TFE in ref. 42, using the Gaussian $03^{48}$ and Antechamber ${ }^{49}$ software packages. Due to the non-equivalence of the carbon-bonded atoms in the fluoromethyl groups of MFE and DFE, the charges were further refined by extracting conformations of either alcohol every $0.1 \mathrm{~ns}$ of a $25 \mathrm{~ns}$ simulation of the alcohol in water (following the simulation protocol described below). A RESP fit was conducted for each of these structures and the final partial atomic charges of MFE and DFE result from the mean charges over all structures. ACPYPE ${ }^{50}$ was used to convert topology files from $\mathrm{AMBER}^{51}$ into Gromacs ${ }^{52-58}$ -readable format.

The initial simulation boxes were assembled with the tools available in the Gromacs software package. Each box was minimized first with the steepest-descent and subsequently with the L-BFGS algorithms. Equilibration was done for $100 \mathrm{ps}$ in the NVT ensemble followed by $100 \mathrm{ps}$ in the $N p T$ ensemble. These simulation times are sufficient to equilibrate the volume of the box and the hydration shell of the solute (results not shown). The equilibrated boxes were then simulated for $2 \mathrm{~ns}$ in the $N p T$ ensemble, and a frame whose volume was nearest to the average box volume in the simulation was collected. This frame was used as the starting configuration in a $25 \mathrm{~ns} N V T$ simulation, with a leapfrog integration scheme, a time-step of 2 fs, and sample collection every $0.1 \mathrm{ps}$. Langevin dynamics with a $0.05 \mathrm{ps}^{-1}$ collision frequency were used to minimize perturbations of the system's dynamics. This procedure, from system equilibration to production run, was repeated for each temperature $(278 \mathrm{~K}, 298 \mathrm{~K}$, $318 \mathrm{~K}, 338 \mathrm{~K}, 358 \mathrm{~K})$. Coulombic and van der Waals interactions are cut-off at $1.2 \mathrm{~nm}$, the latter being additionally switched to zero between 1.0 and $1.2 \mathrm{~nm}$. PME $^{59}$ summation was used in calculating long-range electrostatics, with a fourth-order interpolation and $0.1 \mathrm{~nm}$ grid spacing. Long-range dispersion corrections were used in the calculation of pressure and energy. LINCS $^{60}$ constraints were applied to bonds involving hydrogen atoms. Free energies of hydration were calculated with free energy perturbation (FEP) and the BAR method, as described in a previous study. ${ }^{42}$ In short, a total of 80 steps were used in decoupling coulombic (21 evenly spaced steps) and Lennard-Jones (59 unevenly spaced steps) interactions. Each decoupling simulation ran for $5 \mathrm{~ns}$, after a four-step equilibration protocol as described above, at $298 \mathrm{~K}$, and the phase-space overlap of adjacent decoupling steps was confirmed to be sufficient.

All simulations were carried with Gromacs; statistics on the formation of non-water-bonded structures were collected with $\mathrm{VMD}^{61}$ (in-house scripts).

\section{Symmetry-adapted perturbation theory}

Symmetry-adapted perturbation theory (SAPT) calculations were carried with the Psi4 software package ${ }^{62}$ at the DF-SAPT2 + (CCD)SMP2 level of theory. ${ }^{63-68}$ Non-valence electrons are treated under the frozen core approximation, a density-fitting approach ${ }^{69,70}$ is applied to two-electron integrals in self-consistent field calculations and a superposition of atomic densities is used to obtain the initial density-fitted orbitals. The aug-cc-pVTZ ${ }^{71-74}$ basis sets were used throughout all calculations. This methodology was ranked as the topmost level of theory for SAPT calculations. ${ }^{68}$ SAPT was used to quantify and decompose the interaction energy between one alcohol molecule and one water molecule, in configurations selected from MD simulations of a single solute in a water box at $298 \mathrm{~K}$. The intermolecular interactions were evaluated in two types of configurations: those where the water molecule forms a hydrogen bond-like structure with the solute according to the criteria outlined above, and, for comparison, those where this structure does not exist. In selecting which structures are submitted to a SAPT calculation, we impose also the criterium that the water oxygen must be beyond $4.5 \AA$ of the carbon atom containing the alcohol function, so that only structures with 'direct' interactions between water and the fluorinated methyl groups are reported.

\section{Molecular dynamics with potentials from density functional theory}

We performed first principles molecular dynamics simulations of MFE, DFE and TFE in water. ${ }^{75}$ A snap-shot of the MD simulations with classical force fields of TFE in water ${ }^{42}$ was used to prepare the starting configuration for first-principles simulations. The cubic box was trimmed down to a side length of $1.4 \mathrm{~nm}$, containing the alcohol and 125 water molecules. To build the models for DFE and MFE, one or two fluorine atoms were replaced by hydrogen atoms, respectively. One water molecule situated in a position close to the fluorinated methyl group was selected to represent a hydrogen bond-like structure. This conformation was enforced by restraining the distance between the water oxygen atom and the fluorine atom of the methyl group to $2.6 \AA$ (MFE), $2.8 \AA$ (DFE) and $2.9 \AA$ (TFE) with a force constant of $100 \mathrm{kcal} \mathrm{mol}^{-1} a_{0}{ }^{-2}$ (where $a_{0}$ is the Bohr radius), while keeping the $\mathrm{O}-\mathrm{H}-\mathrm{F} / \mathrm{H}$ angle restrained to $170^{\circ}$ with a force constant of $100 \mathrm{kcal} \mathrm{mol}^{-1} \mathrm{rad}^{-2}$. The above distances correspond to the mean value of the oxygen-fluorine distance in 2890 (MFE), 1537 (DFE) and 1314 (TFE) configurations of water-solute hydrogen bond-like structures extracted from MD simulations at $298 \mathrm{~K}$.

For all alcohol systems, a 20 ps long simulation was performed using the Quickstep module ${ }^{76}$ of the CP2K package ${ }^{77}$ which is based on the Gaussian and plane waves method (GPW) to represent the wavefunction and its augmented extension (GAPW) to describe the electron density. ${ }^{78}$ We used double zeta valence plus (DZVP) basis set. The interaction between valence and core electrons is described by Geodecker-Teter-Hutter (GTH) norm conserving pseudopotentials. A plane wave expansion for the charge density is employed using an energy cutoff of 500 Ry. The BLYP functional is used as exchange correlation functional and the empirical pair potential in Grimme's D3 method $^{79}$ was employed for dispersion correction. The equations of motion were integrated with a 0.5 fs time resolution. Atom positions were saved every time-step. From the trajectories, the coordinates of the restrained water molecule were extracted and 
power spectra for the OH-group facing the fluorinated methyl group were calculated using the TRAVIS ${ }^{80}$ program. All spectra plots were generated with the matplotlib.pyplot module in Python. ${ }^{81}$

\section{Results}

\section{Raman-MCR spectra}

Fig. 2A shows unprocessed Raman spectra obtained at $20^{\circ} \mathrm{C}$ for $1 \mathrm{~mol} \mathrm{dm}^{-3}$ solutions of MFE, DFE, and TFE, and pure water. A slightly higher background is present in the spectrum of MFE due to fluorescence as this sample is less pure than the other two fluorinated alcohol samples. We note that this level of interfering fluorescence is not detrimental to our analysis given that it can be effectively removed using a linear (or quadratic) background subtraction to produce the SC spectra shown in Fig. 2B (and the associated impurity concentration is far below that of the solute species). The latter SC spectra were obtained using SMCR, from pairs of pure water and solution spectra shown in Fig. 2A. These (unnormalized) SC component spectra clearly indicate a difference in the $\mathrm{CH}$ stretch band intensity reflecting the different number of $\mathrm{CH}$ groups in each one of the fluorinated alcohols $\left(n_{\mathrm{CH}}=4\right.$ in MFE, $n_{\mathrm{CH}}=3$ in DFE, and $n_{\mathrm{CH}}=2$ in TFE). Additionally, as highlighted in the inset panel in Fig. 2B, these SC spectra also display vibrational features arising from water molecules whose $\mathrm{OH}$ band is perturbed by the solute and thus differ from the $\mathrm{OH}$ band of pure water. All three fluorinated alcohols exhibit a prominent and relatively narrow $\mathrm{OH}$ feature with a frequency near $3660 \mathrm{~cm}^{-1}$, which has previously been assigned to water dangling $\mathrm{OH}$ defects in the hydrogen-bonded hydration-shell structure. ${ }^{42}$ For all fluorinated solutes, the dangling $\mathrm{OH}$ feature is significantly more intense than the previously measured dangling $\mathrm{OH}$ in the hydration-shell of $\mathrm{EtOH}^{43}$ (as previously shown ${ }^{42}$ ). More interestingly, the intensities of the dangling $\mathrm{OH}$ peaks do not increase in proportion to the number of fluorine atom substitutions, suggesting that the dangling $\mathrm{OH}$ probability around the single fluorine atom in
MFE is larger than that for each of the three fluorine atoms in TFE (as further discussed and quantified below). Moreover, the vibrational frequencies of the dangling $\mathrm{OH}$ peaks are significantly redshifted for MFE $\left(3626 \mathrm{~cm}^{-1}\right)$ and DFE $\left(3656 \mathrm{~cm}^{-1}\right.$ ), relative to TFE and $\mathrm{EtOH}$, both of which have dangling $\mathrm{OH}$ frequencies near $3670 \mathrm{~cm}^{-1} .^{42}$

\section{Thermodynamics of dangling hydroxy formation}

Fig. 3 shows the temperature dependent Raman-MCR SC $\mathrm{CH}$ and hydration-shell $\mathrm{OH}$ spectra of the three fluorinated ethanol solutes along with the spectra of pure water. These results clearly reveal that the hydration-shells of MFE have a significantly weaker temperature dependence than the hydration-shells of DFE and TFE. Our goal is to use these spectra to obtain the corresponding thermodynamics of forming the dangling $\mathrm{OH}$ defect structure around all three fluorinated ethanol solutes from a hydrationshell that contains no dangling $\mathrm{OH}$ defects.

Since the intensity of the dangling $\mathrm{OH}$ band is proportional to the probability that such a structure will be found in the hydration shell, its temperature dependence may be used to obtain the Gibbs energy $(\Delta G)$, enthalpy $(\Delta H)$, and entropy $(\Delta S)$ of formation of these defects. However, obtaining the dangling $\mathrm{OH}$ band intensity requires subtracting the background intensity underlying the dangling $\mathrm{OH}$ band. Since the shape of the background is not precisely known, the background subtraction procedure is the most significant source of error in the resulting $\Delta G, \Delta H$ and $\Delta S$ values. We have determined that the background can be reasonably well approximated by either a Gaussian or cubic polynomial function fit to the points on either side of the dangling $\mathrm{OH}$ band of interest. The following results pertain to the average dangling $\mathrm{OH}$ band intensities obtained using the two background fitting procedures, and the associated error bars reflect the difference between the band areas obtained in these two ways (see ESI, $\dagger$ for further details).

Fig. 4 shows the dangling $\mathrm{OH}$ peaks (solid curves) obtained after subtracting the cubic background (dashed curves) from
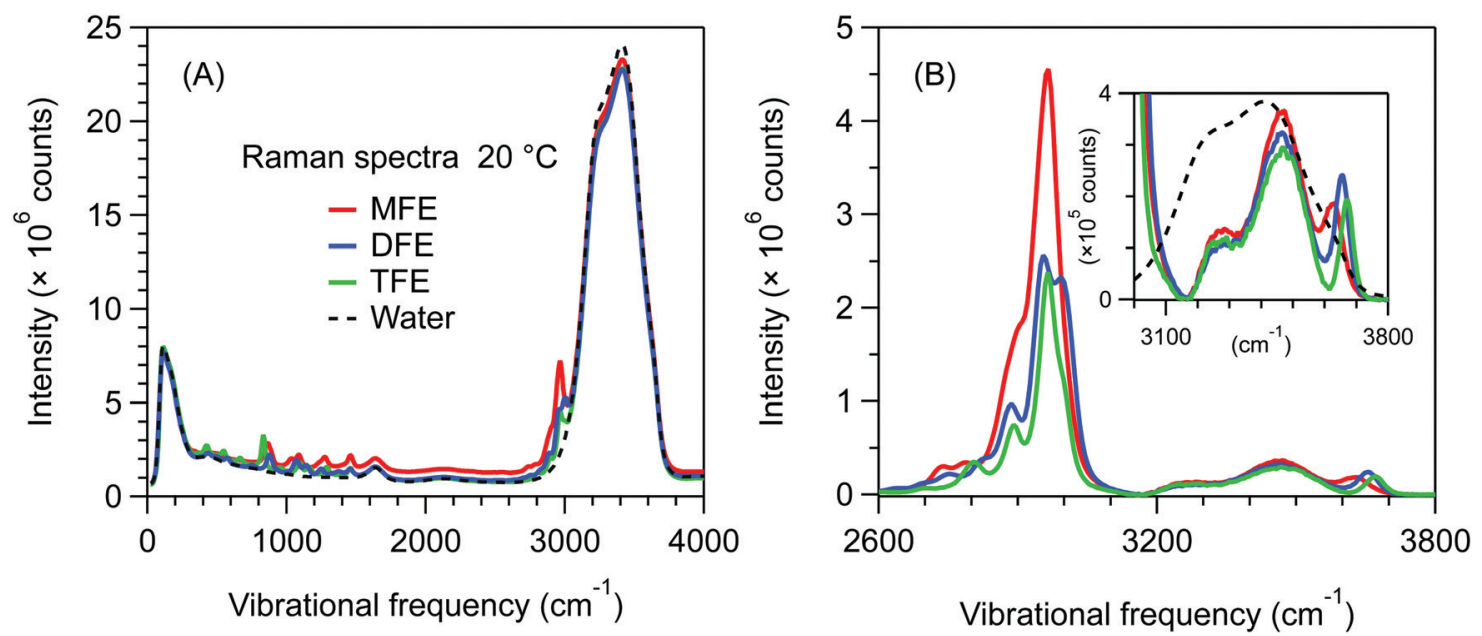

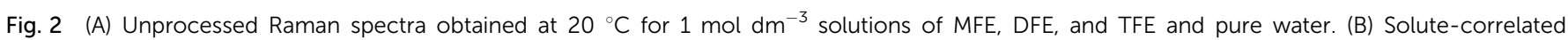

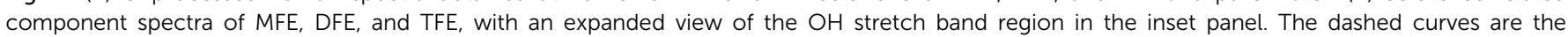
corresponding pure water Raman spectra, arbitrarily scaled to highlight the difference in shape between the pure water and SC OH stretch bands. 

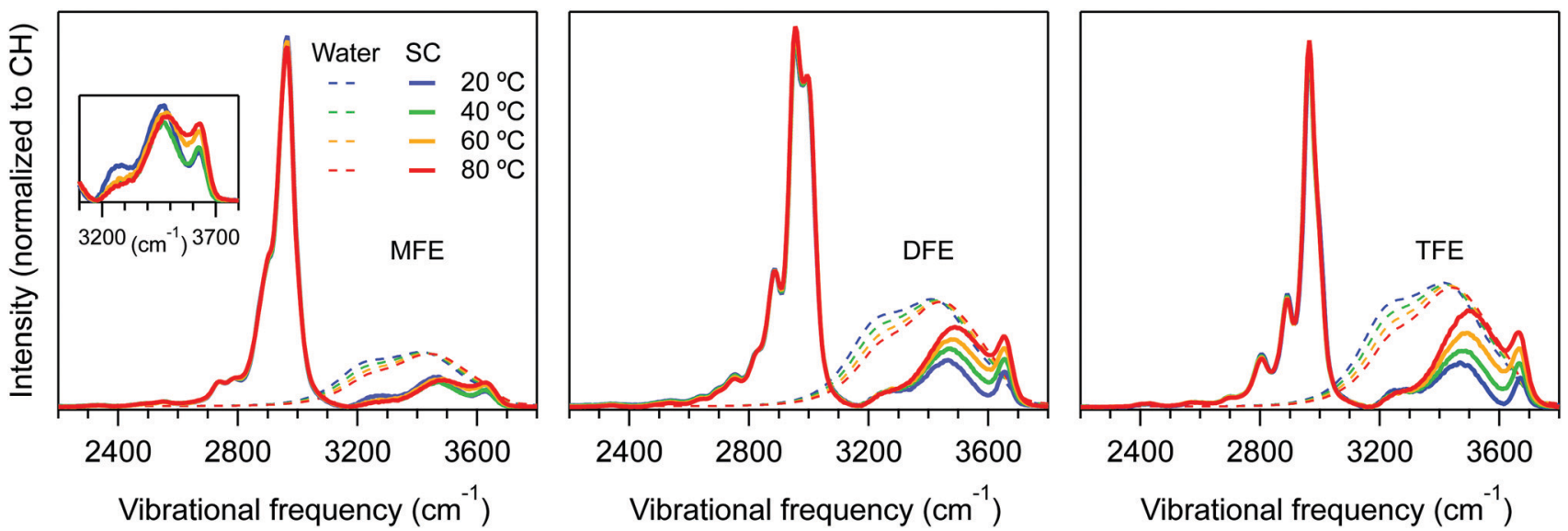

Fig. 3 Hydration-shell spectra of MFE, DFE, and TFE normalized to the $\mathrm{CH}$ band area. The solid curves represent the solute-correlated (SC) spectra and the dashed curves represent the (arbitrarily scaled) spectra of pure water.
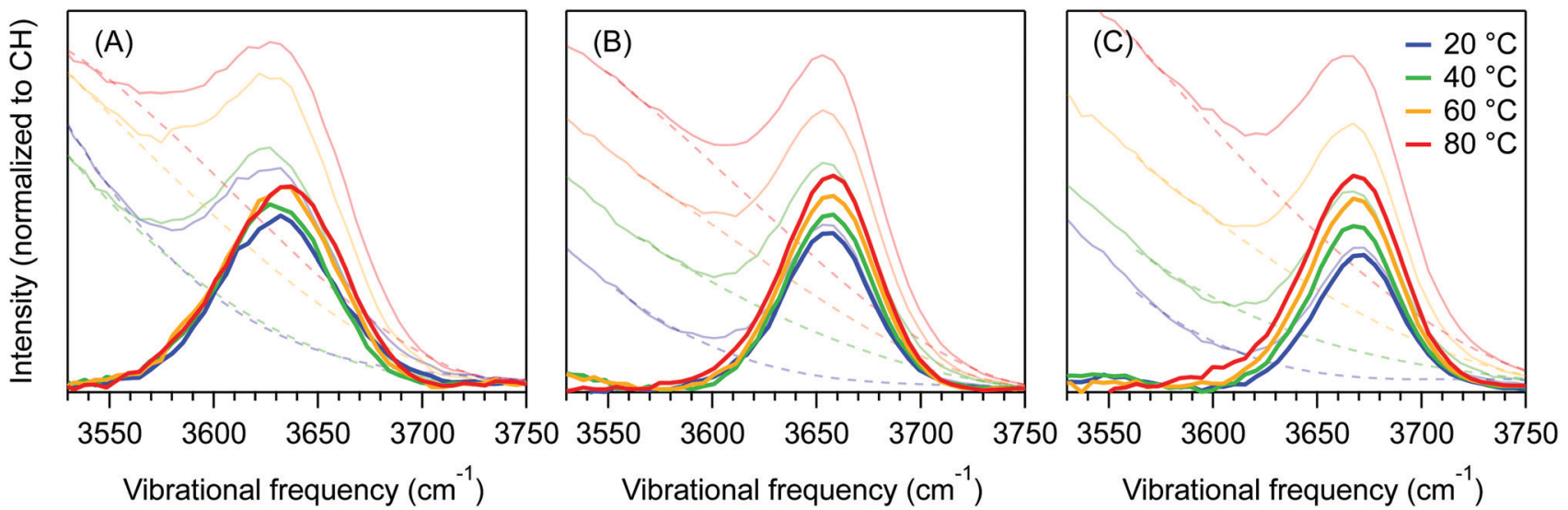

Fig. 4 Temperature dependence of the dangling OH peaks in the hydration shell of (A) MFE, (B) DFE and (C) TFE. These peaks were obtained from the respective minimum area non-negative SC spectra (transparent solid curves), after subtracting the background (dashed curves) using a cubic polynomial function.

the SC hydration-shell spectra (transparent solid curves). The corresponding results obtained assuming a Gaussian background shape are shown in ESI, $\dagger$ Fig. S1. The areas of these dangling $\mathrm{OH}$ peaks yield $\langle k\rangle$, which is the corresponding experimentally determined average number of dangling $\mathrm{OH}$ defects in the solute hydration-shell, whose values are plotted in Fig. 5 .

Fig. 6 shows the $\Delta G, \Delta H$ and $\Delta S$ values obtained from the experimental values of $\langle k\rangle$. As can be seen, the enthalpy of forming a dangling $\mathrm{OH}$ structure is invariably positive, but increases in magnitude with increasing number of fluorine atoms on the solute. This implies that the $\mathrm{OH} \cdots \mathrm{F}$ interaction is invariably less enthalpically favorable than a water $\mathrm{OH} \cdots \mathrm{O}$ hydrogen-bonded structure, but the $\mathrm{OH} \cdots \mathrm{F}$ interaction is more enthalpically favorable for MFE than for the other two solutes. Specifically, if we assume that the previously measured enthalpy of forming a dangling $\mathrm{OH}$ around $\mathrm{EtOH}\left(\Delta H \approx 14 \mathrm{~kJ} \mathrm{~mol}^{-142,43}\right)$ roughly corresponds to that of breaking a water-water hydrogen bond, then the above $\Delta H$ values provide the following estimates of the enthalpy of the $\mathrm{OH} \cdots \mathrm{F}$ hydrogen bond: $\Delta H_{\mathrm{TFE}} \approx$ $12-14=-2 \mathrm{~kJ} \mathrm{~mol}^{-1}, \Delta H_{\mathrm{DFE}} \approx 8-14=-6 \mathrm{~kJ} \mathrm{~mol}^{-1}$, and $\Delta H_{\mathrm{MFE}} \approx 3-14=-11 \mathrm{~kJ} \mathrm{~mol}^{-1}$.

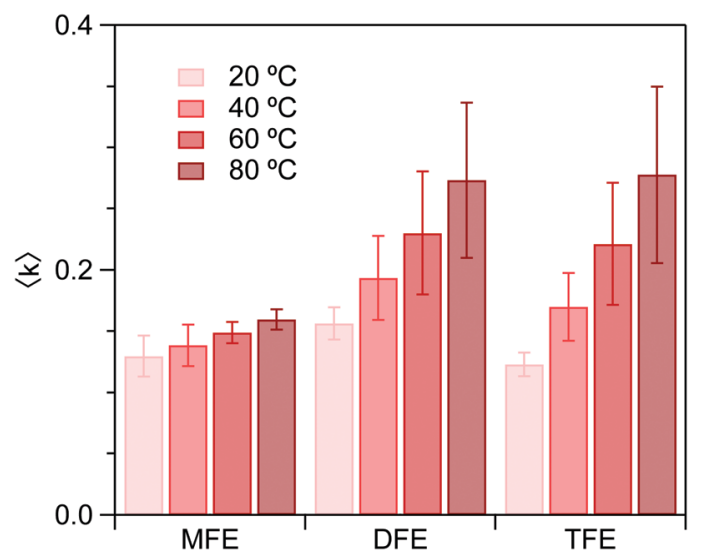

Fig. 5 Experimental average number, $\langle k\rangle$, of dangling $\mathrm{OH}$ structures per hydration shell, determined using the high-frequency $\mathrm{OH}$ peak areas.

The results in Fig. 6 further reveal that the entropy change associated with forming a dangling $\mathrm{OH}$ decreases with the number of fluorine atoms on the solute. Moreover, the dangling $\mathrm{OH}$ formation $\Delta S$ is positive for TFE and DFE but becomes 

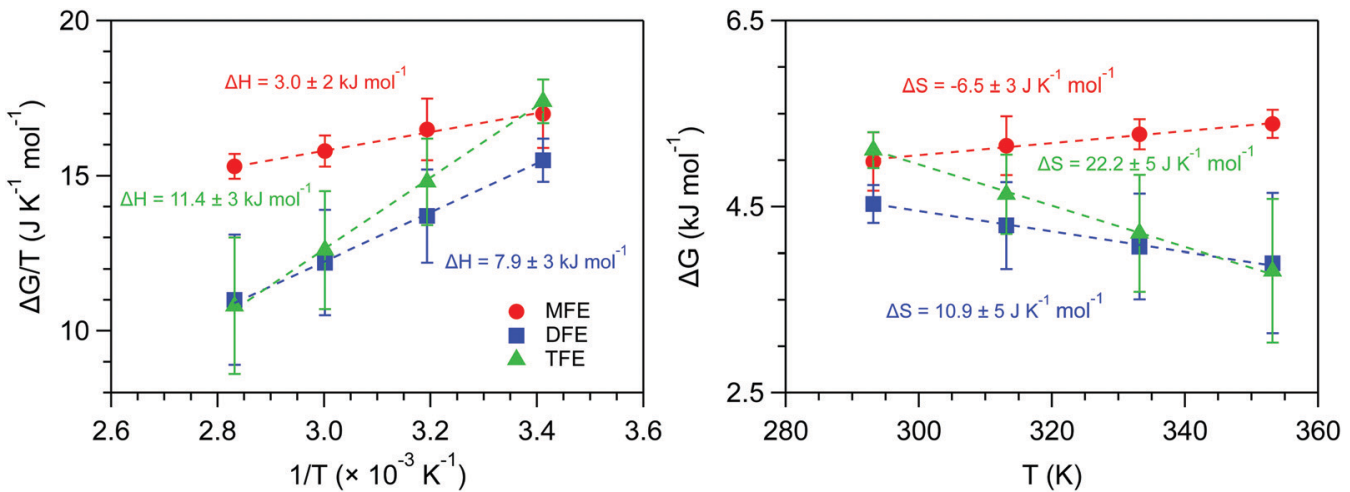

Fig. 6 Experimental enthalpy and entropy changes associated with the formation of dangling $\mathrm{OH}$ structures with increasing fluorination of the methyl group of ethanol, obtained using the experimental average $\langle k\rangle$ values.

negative for MFE. This implies that the dangling $\mathrm{OH}$ species around TFE and DFE are more flexible, while that around MFE is less flexible, relative to a water-water hydrogen-bond. Thus, both the $\Delta H$ and $\Delta S$ results imply that the single fluorine atom in MFE forms a relatively strong and rigid dangling $\mathrm{OH}$ structure, and thus may reasonably be described as an $\mathrm{OH} \cdots \mathrm{F}$ hydrogen bond, while adding more fluorine atoms to the terminal methyl group of ethanol weakens the associated $\mathrm{OH} \cdots$ F structures and increases their flexibility.

\section{The experimentally-detected dangling hydroxy band arises from non-water-bonded hydroxy groups}

Molecular dynamics (MD) simulations were used, together with eqn (4), to determine the temperature dependence of the formation of non-water-bonded hydroxy structures in the hydration shell of the solutes. As mentioned above (Fig. 1), this population actually comprises two subpopulations: hydroxy groups pointing to the tail of the solute (called hydrogen bond-like structures) and free hydroxys. In Fig. 7(A) we quantify the non-water-bonded hydroxy structure population; the hydrogen bond-like subpopulation is shown in panel (B) of the same figure. Comparing these results with experiment (Fig. 5) clarifies that the population, in the MD simulations, that best represents the spectroscopically active population giving rise to the dangling
$\mathrm{OH}$ peak from experiment is that of non-water-bonded hydroxy groups, that is, both free hydroxy groups and hydrogen bond-like hydroxy groups pointing towards the solute.

This conclusion is further supported by comparing the thermodynamics of formation of non-water-bonded hydroxy structures obtained with simulation (Fig. 8) and experiment (Fig. 6). Even though the absolute values of the enthalpy and entropy of formation differ between experiment and simulation, the observed trends are equivalent. More specifically, the enthalpy required to break a water-water hydrogen bond in the hydration shell of the solute to form either a free hydroxy or a hydrogen-bond-like structure becomes increasingly more unfavourable with increasing fluorine content. Conversely, the entropy change for the same process becomes increasingly more favourable with increasing degree of fluorination and shows a change in sign from MFE to DFE. This change in sign is of particular interest, since it implies that a non-water-bonded structure near a monofluoromethyl group has less conformational freedom than a water-water hydrogen bond in the same region, whereas structures near DFE and TFE are less conformationally restricted. We expect that the qualitative trends shown by the thermodynamic observables calculated from simulation are reliable, despite the fact that these values are necessarily affected by artificial correlations that arise from using periodic
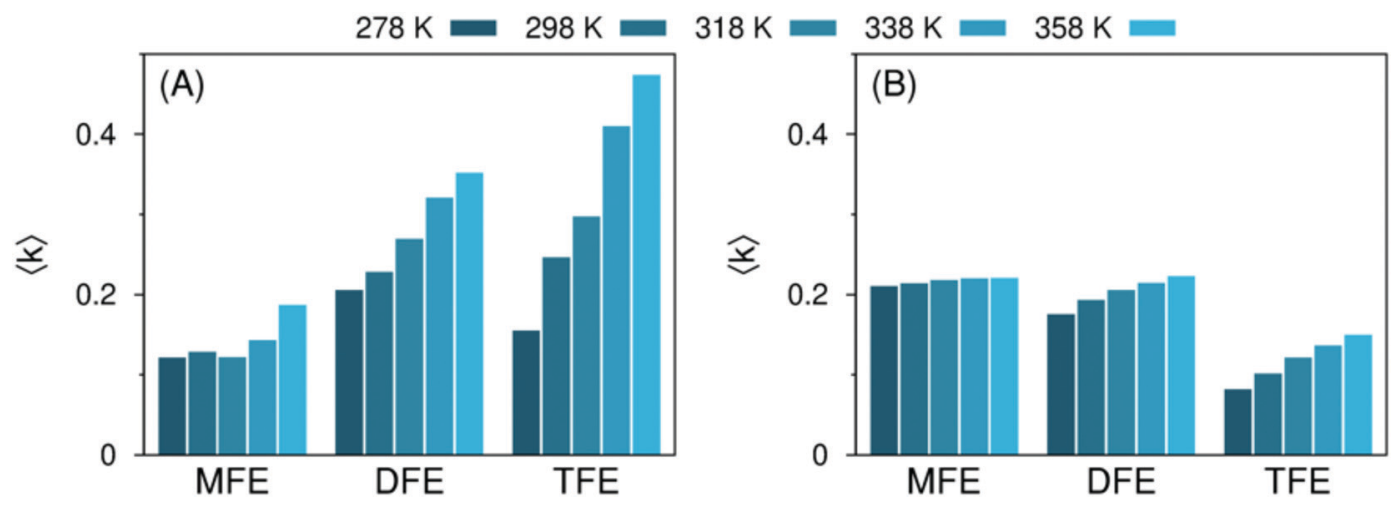

Fig. 7 Predicted average number $\langle k\rangle$ of non-water-bonded (panel A) and hydrogen bond-like (panel B) hydroxy structures per hydration shell, quantified with MD simulations in the $278 \leq T / K \leq 358$ range, for solutions of MFE, DFE and TFE. Data for TFE are obtained from ref. 42 . All data are presented in ESI, $\dagger$ Table S5. 

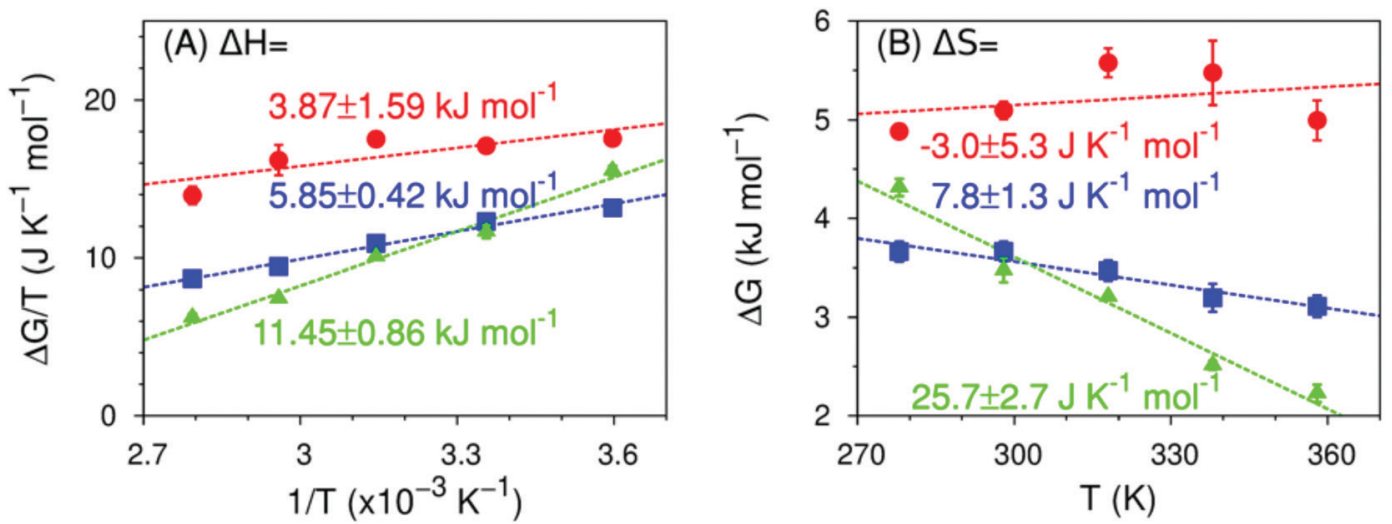

Fig. 8 Predicted enthalpy and entropy of formation of a non-water-bonded hydroxy structures (MFE as red circles, DFE as blue squares, TFE as green triangles) in the $278 \leq T / K \leq 358$ range, calculated from MD simulations using eqn (7), as well as eqn (4) to (6). Dashed lines are linear fits to the data points from which the enthalpy (panel A), the entropy (panel B) and their respective errors are calculated. Data for TFE are taken from ref. 42. See ESI, $\dagger$ Table S6.

boundary conditions. We reduced the magnitude of these effects by choosing a cubic simulation box with edge length at least 10 times larger than the diameter of the simulated molecules. These simulation conditions have been shown to substantially reduce finite-size effects associated with interfacial thermodynamic quantities, even in systems with long range interactions. ${ }^{82-84}$

The hydrogen bond-like hydroxy subpopulation is the largest of the two subpopulations making up non-water-bonded hydroxy structures at most temperatures in simulation, and thus should dominate the experimental signal. This conclusion is also supported by the computed power spectra of a water hydroxy group restrained to form a hydrogen bond-like structure in Fig. 9. The spectra qualitatively reflect the experimentally observed trend for the position of the water $\mathrm{O}-\mathrm{H}$ stretching band between $3600 \mathrm{~cm}^{-1}$ and $3700 \mathrm{~cm}^{-1}$ in Fig. 4: the TFE band shows the highest frequency, followed by DFE and MFE. We do, however, observe a shift in the absolute positions of the bands obtained with experimental and computational methods, such

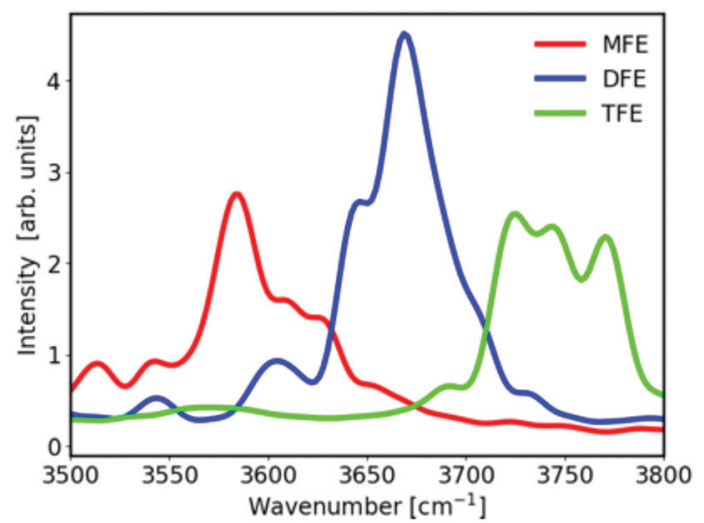

Fig. 9 Predicted power spectra of the water molecule forming a hydrogen bond-like structure with MFE (red), DFE (blue), and TFE (green) computed from first principle simulations of the alcohol molecule in water. The distance between the water molecule oxygen atom and the fluorine atom was restrained to 2.6, 2.8, and $2.9 \AA$ for MFE, DFE, and TFE, respectively. Intensities are unscaled. that the computed location of the corresponding ethanol band (not shown) lies between the DFE and the TFE band, whereas in the experiment the frequencies for dangling hydroxy structures are about the same for ethanol and TFE. ${ }^{42}$ Nonetheless, the fact that the occurrence of hydrogen bond-like structures is sufficient to reproduce the varying band locations observed in the Raman-MCR experiments supports our conclusion that these structures provide a useful means of characterizing the hydration shell of each solute, both experimentally and theoretically.

\section{Hydrogen bonds and dangling hydroxys}

Fig. 10 shows the temperature dependence, calculated using eqn (4), of the formation of hydrogen bond-like structures in the hydration shell of the fluoromethyl groups, such that the hydroxy group points directly at the fluoromethyl moiety. The trends in thermodynamics we have previously observed still hold: the enthalpy becomes more unfavorable with increasing amount of fluorine atoms, the entropy becomes more favorable. However, the absolute values of $\Delta H$ and $\Delta S$ are markedly different from both the experimental values in Fig. 6 and the theoretical values in Fig. 8 (obtained from all the non-waterbonded hydroxy structures): $\Delta H$ ranges from 0 to $6 \mathrm{~kJ} \mathrm{~mol}^{-1}$ and $\Delta S$ from -11 to $3 \mathrm{~kJ} \mathrm{~K}^{-1} \mathrm{~mol}^{-1}$. Since, predominantly, a single such structure is formed at any given time (see ESI, $\dagger$ Fig. S4), the reported free energies follow the balance of attractive water-water and water-fluoromethyl group interactions. The results in Fig. 10 imply that, relative to water-water hydrogen bonds, $\mathrm{CF} \cdots \mathrm{HOH}$ hydrogen-bond like structures in MFE have a similar enthalpy but are more rigid (as they have a more negative entropy). In the case of DFE, the hydrogen bond-like structures are slightly weaker (enthalpically) than water-water hydrogen bonds and again moderately more rigid, while those to TFE are much weaker compared to water-water hydrogen bonds, and slightly more flexible. In other words, water molecules can form hydrogen bonds with the fluorine atom in MFE, dangling hydroxys near the fluorine atoms in TFE and something in between for DFE. 

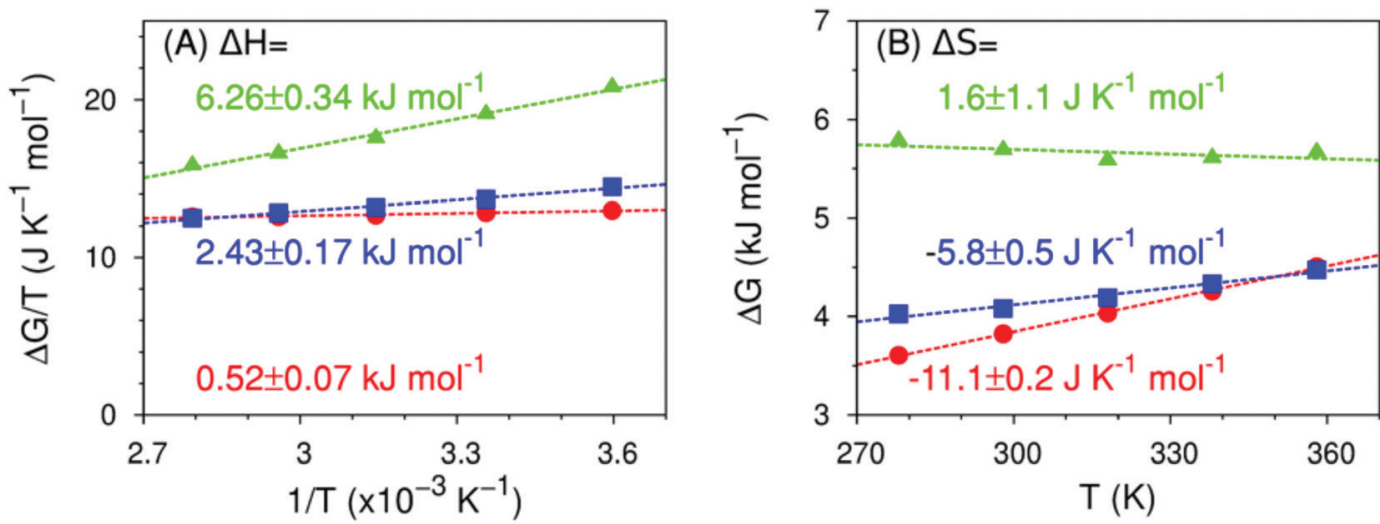

Fig. 10 Predicted enthalpy and entropy of formation of a hydrogen bond-like structure (MFE as red circles, DFE as blue squares, TFE as green triangles) in the $278 \leq T / K \leq 358 \mathrm{~K}$ range, calculated from MD simulations using eqn (4) to (6). Dashed lines are linear fits to the data points from which the enthalpy (panel A), the entropy (panel B) and their respective errors are calculated. Data for TFE are extracted from ref. 42 . Refer to ESI, $\dagger$ Table S6.

\section{A stepwise transition from hydrophilic to hydrophobic}

Given the above results, it is tempting to label $-\mathrm{CFH}_{2}$ as hydrophilic and $-\mathrm{CF}_{3}$ as hydrophobic. We emphasize, however, that differences in the hydrophobicity of these groups will also indirectly reflect different water-water interactions in their hydration shells, which we do not explore here. ${ }^{85}$ To test the validity of these labels we have calculated hydration free energies $\left(\Delta G_{\mathrm{Hyd}}\right)$ of ethanol (EtOH), MFE, DFE and TFE at $298 \mathrm{~K}$ from available experimental data (see ESI, $\dagger$ Tables S1 and S2). The hydration free energy varies as MFE $<$ DFE $<$ EtOH $<$ TFE. Importantly, our simulations (see ESI, $\dagger$ Section S2) show that the dominant contribution to this trend indeed comes from the tail of the alcohols, indicating that the labels given to mono-, di- and trifluorinated groups are appropriate.

\section{Discrete, attractive, solute-water interactions of hydrophobes and hydrophiles}

Direct solute-water interactions can be quantified using symmetry-adapted perturbation theory calculations, the results of which are shown in Fig. 11A and ESI, $\dagger$ Fig. S5A for several water-solute dimer configurations in vacuum. These configurations were extracted from MD simulations of the solute in a water box, and are therefore representative of a fully hydrated solute. The variation of the total interaction energy between a solute and a single water molecule along the distance between the water oxygen atom and the solute's closest fluorine atom is distinct between MFE, DFE or TFE only when the water molecule forms a hydrogen bond-like structure with the solute. For these configurations, the interaction becomes less favorable with increasing number of fluorine atoms (Fig. 11A). The observed trend in stability is in agreement with the enthalpies of formation of hydrogen bond-like structures in Fig. 6, 8 and 10. When the water molecule does not form such a structure, the mean value of the interaction energy distributions is close to zero for the 3 alcohols (ESI, $\dagger$ Fig. S5A).

Conveniently, SAPT calculations allow us to decompose the total interaction energy into electrostatics, London dispersion, induction and exchange contributions, shown as averages in
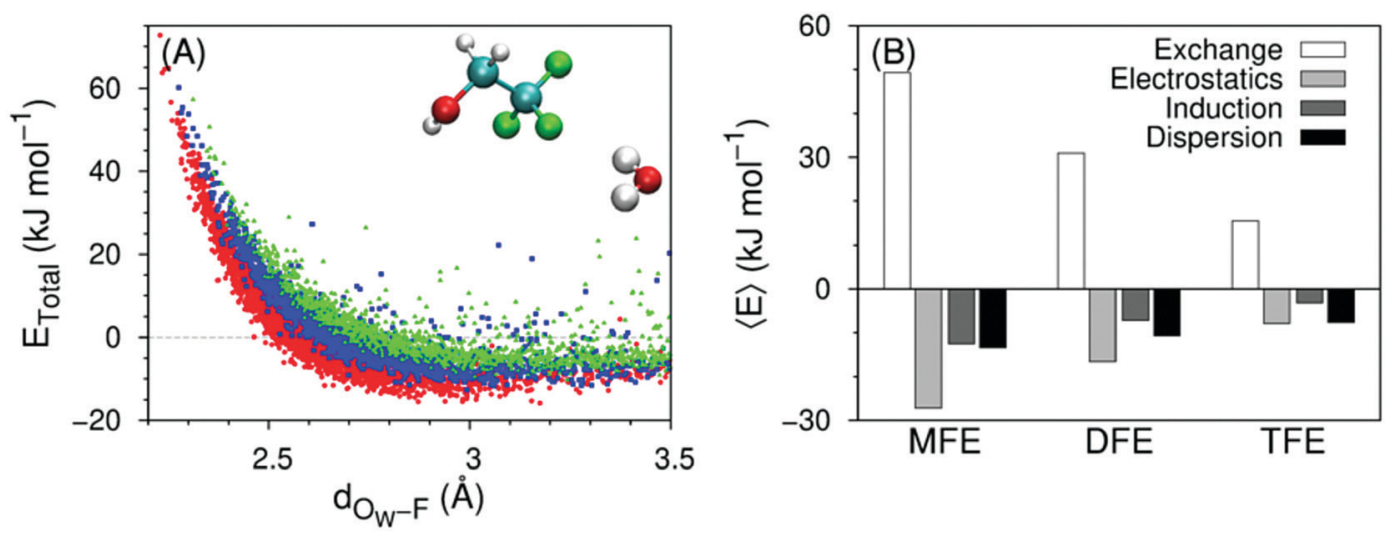

Fig. 11 (A) Total interaction energy vs. distance for water-solute dimers forming a hydrogen bond-like structure (water-MFE as red circles, water-DFE as blue squares, water-TFE as green triangles), calculated at the DF-SAPT2 + (CCD)8MP2/aug-cc-pVTZ level of theory. The distance corresponds to the closest water oxygen-fluorine pair. Configurations are extracted from MD simulations at $298 \mathrm{~K}$. The inset molecules represent a conformation of a water-TFE dimer corresponding to one of the plotted data points. (B) Average energy components $\langle E\rangle$, calculated for distances above that where the total energy becomes attractive $(2.5,2.6$ and $2.8 \AA$ for MFE, DFE and TFE, respectively). Data for TFE are taken from ref. 42 . The number of points in each data set is MFE: 2890; DFE: 1537; TFE: 1314. Related with Fig. S5, S6 and Table S8 (ESI†). 
Fig. 11B (the dependence of the different contributions on waterfluorine distance is collected in ESI, $\dagger$ Fig. S6). All contributions decrease in magnitude with increasing degree of fluorination. However, the ratio of different attractive contributions (ESI, $\dagger$ Fig. S5B) is more informative: whereas electrostatics is always twice as large as induction, the dispersion:induction ratio increases from one to two along the transition from MFE to TFE and the dispersion:electrostatics ratio increases from one half to one.

\section{Concluding remarks}

We have investigated the sequential fluorination of a methyl group (in ethanol) and the associated changes in hydrophobicity, hydration shell structure and thermodynamics. The hydrophobicity of a methyl group scales non-monotonically with the degree of fluorination, as the hydrophobicity of a methyl group initially decreases and then increases with the addition of 1 to 3 fluorine atoms. We have shown that a specific hydration shell structure, $\mathrm{CF} \cdot \mathrm{HOH}$, meeting geometric criteria commonly used to define water-solute hydrogen bonds, is present in aqueous solutions of each of these alcohols. Further, the free energy, enthalpy and entropy of formation of this structure, which are experimentally accessible using spectroscopic methods, are sufficiently different as to allow for a categorization of the structures into either hydrogen bond (MFE) or dangling hydroxy (TFE), or a structure exhibiting intermediate spectroscopic and thermodynamics features (DFE). Moreover, both enthalpy and entropy are indispensable for our understanding of hydrogen bonds. The hydrogen bond donated by water to the fluorine in MFE actually has comparable enthalpy to that of water-water hydrogen bonds; the former is overall much weaker than the later only because of its high entropic cost. Finally, the balance of attractive solutewater interactions, namely London dispersion and electrostatics, closely follows the categorization: hydrogen bonds are highly directional owing to a large electrostatic stabilization from the aligned dipoles of the $\mathrm{C}-\mathrm{F}$ and the $\mathrm{H}-\mathrm{O}$ bonds; loosely bonded dangling hydroxys have a much weaker stabilizing contribution of electrostatics, which is near-exactly matched by London dispersion. Our results demonstrate that the combined use of RamanMCR and theoretical calculations may be used to quantify and mechanistically explain subtle changes in hydrogen bond character and local hydrophobicity associated with sequential fluorination of a methyl group, and suggest that the same strategy could be extended to other solute-solvent interactions.

The effects of mono-, di- and trifluorination on solute-water interactions reported here and, more generally, the importance of understanding the entropic and enthalpic signature of solute-water hydrogen bonds (relative to water-water hydrogen bonds in the bulk liquid) have potentially important implications for drug design and for our understanding of biological systems. This importance is illustrated by the surprising capability of fluorinated BPTI derivatives to inhibit $\beta$-trypsin. The $\beta$-trypsinBPTI binding pocket contains up to five water molecules, which interact with nearby amino acids and play a role in the binding affinity. Wild-type BPTI has an arginine amino acid in the binding loop. Replacing arginine by ethylglycine dramatically reduces BPTI's inhibitory capacity-unsurprising, given that the long, positively charged side chain of arginine was replaced by a short and electrostatically neutral one $\left(\mathrm{CH}_{2} \mathrm{CH}_{3}\right)$. Replacing the methyl group in ethylglycine by di- or trifluorinated variants, however, recovers BPTI's inhibitory capacity to wild-type levels. ${ }^{23}$ Our results point towards a mechanistic explanation of these changes.

Predicting whether two molecules interact mainly through electrostatics or dispersion is a long-standing challenge, and answering it typically requires a combination of methods which have limited application in condensed phase systems. ${ }^{86-88}$ Here we have demonstrated that hydration shell spectroscopy accompanied by simulation can be used to elucidate such interactions. Increasing enthalpic cost and entropic gain of forming hydrogen bond-like structures does correlate with a decrease in the electrostatics: dispersion ratio for ethanol, MFE, DFE and TFE. ${ }^{42}$ Thus it is possible to link electrostatic and dispersive contributions to the formation of different solute-water hydrogen bond-like structures and their influence on hydration thermodynamics.

\section{Author contributions}

J. R. R. performed classical simulations, SAPT calculations; D. M. O. performed experiments; P. I. performed first principles simulations; D. B. A. supervised experiments; A. V. V. supervised classical simulations and designed initial project. All authors contributed to project design, analyses and writing.

\section{Conflicts of interest}

There are no conflicts of interest to declare.

\section{Acknowledgements}

Support for D. B.-A. and D. M. O. was provided by the US National Science Foundation (CHE-1763581) and Purdue University. J. R. R., P. I. and A. V. V. acknowledge support by the Deutsche Forschungsgemeinschaft (SPP 1807). Computational resources provided by the North-German Supercomputing Alliance (HLRN) and by the ZEDAT at Freie Universität Berlin are gratefully acknowledged. Open Access funding provided by the Max Planck Society.

\section{References}

1 A. J. Patel, P. Varilly, S. N. Jamadagni, M. F. Hagan, D. Chandler and S. Garde, J. Phys. Chem. B, 2012, 116, 2498-2503.

2 P. Ball, Chem. Rev., 2008, 108, 74-108.

3 M.-C. Bellissent-Funel, A. Hassanali, M. Havenith, R. Henchman, P. Pohl, F. Sterpone, D. van der Spoel, Y. Xu and A. E. Garcia, Chem. Rev., 2016, 116, 7673-7697.

4 D. Chandler, Nature, 2005, 437, 640.

5 D. Ben-Amotz, Annu. Rev. Phys. Chem., 2016, 67, 617-638. 
6 G. Schirò, Y. Fichou, F.-X. Gallat, K. Wood, F. Gabel, M. Moulin, M. Härtlein, M. Heyden, J.-P. Colletier and A. Orecchini, et al., Nat. Commun., 2015, 6, 6490.

7 P. Anilkumar, T. B. Lawson, S. Abbina, J. T. A. Mäkelä, R. C. Sabatelle, L. E. Takeuchi, B. D. Snyder, M. W. Grinstaff and J. N. Kizhakkedathu, Nat. Commun., 2020, 11, 2139.

8 D. I. Fried, D. Bednarski, M. Dreifke, F. J. Brieler, M. Thommes and M. Fröba, J. Mater. Chem. B, 2015, 3, 2341-2349.

9 G.-P. Hao, G. Mondin, Z. Zheng, T. Biemelt, S. Klosz, R. Schubel, A. Eychmüller and S. Kaskel, Angew. Chem., Int. Ed., 2015, 54, 1941-1945.

10 I. K. Ilic, M. Perovic and C. Liedel, ChemSusChem, 2020, 13, 1856-1863.

11 V. Sahu, S. Shekhar, P. Ahuja, G. Gupta, S. K. Singh, R. K. Sharma and G. Singh, RSC Adv., 2013, 3, 3917-3924.

12 Y.-T. Kim and T. Mitani, J. Power Sources, 2006, 158, 1517-1522.

13 L. Kou and C. Gao, Nanoscale, 2013, 5, 4370-4378.

14 A. Wittkopp and P. R. Schreiner, Chem. - Eur. J., 2003, 9, 407-414.

15 J. J. Gajewski, Acc. Chem. Res., 1997, 30, 219-225.

16 J. B. F. N. Engberts and M. J. Blandamer, Chem. Commun., 2001, 1701-1708.

17 Y. Luo, H. Ma, S. Zhang, D. Zheng, P. Che, X. Liu, M. Zhang, J. Gao and J. Xu, J. Am. Chem. Soc., 2020, 142, 6085-6092.

18 Q. Xiao, M. Delbianco, S. E. Sherman, A. M. Reveron Perez, P. Bharate, A. Pardo-Vargas, C. Rodriguez-Emmenegger, N. Y. Kostina, K. Rahimi, D. Söder, M. Möller, M. L. Klein, P. H. Seeberger and V. Percec, Proc. Natl. Acad. Sci. U. S. A., 2020, 117, 11931-11939.

19 T. Kawasaki, M. Tokuhiro, N. Kimizuka and T. Kunitake, J. Am. Chem. Soc., 2001, 123, 6792-6800.

20 S. E. Boyken, Z. Chen, B. Groves, R. A. Langan, G. Oberdorfer, A. Ford, J. M. Gilmore, C. Xu, F. DiMaio, J. H. Pereira, B. Sankaran, G. Seelig, P. H. Zwart and D. Baker, Science, 2016, 352, 680-687.

21 J. R. Robalo and A. Vila Verde, Phys. Chem. Chem. Phys., 2019, 2029-2038.

22 E. Camerino, D. M. Wong, F. Tong, F. Körber, A. D. Gross, R. Islam, E. Viayna, J. M. Mutunga, J. Li and M. M. Totrov, et al., Bioorg. Med. Chem. Lett., 2015, 25, 4405-4411.

23 S. Ye, B. Loll, A. A. Berger, U. Mülow, C. Alings, M. C. Wahl and B. Koksch, Chem. Sci., 2015, 6, 5246-5254.

24 B. Zheng, S. V. D'Andrea, L.-Q. Sun, A. X. Wang, Y. Chen, P. Hrnciar, J. Friborg, P. Falk, D. Hernandez, F. Yu, A. K. Sheaffer, J. O. Knipe, K. Mosure, R. Rajamani, A. C. Good, K. Kish, J. Tredup, H. E. Klei, M. Paruchuri, A. Ng, Q. Gao, R. A. Rampulla, A. Mathur, N. A. Meanwell, F. McPhee and P. M. Scola, ACS Med. Chem. Lett., 2018, 9, 143-148.

25 A. A. Berger, J. S. Völler, N. Budisa and B. Koksch, Acc. Chem. Res., 2017, 50, 2093-2103.

26 E. N. G. Marsh, Acc. Chem. Res., 2014, 47, 2878-2886.

27 B. C. Buer, B. J. Levin and E. N. G. Marsh, J. Am. Chem. Soc., 2012, 134, 13027-13034.

28 M. Salwiczek, E. K. Nyakatura, U. I. M. Gerling, S. Ye and B. Koksch, Chem. Soc. Rev., 2012, 41, 2135-2171.

29 D. Giménez, C. Andreu, M. del Olmo, T. Varea, D. Diaz and G. Asensio, Bioorg. Med. Chem., 2006, 14, 6971-6978.
30 H. Meng and K. Kumar, J. Am. Chem. Soc., 2007, 129, 15615-15622. 31 Y. Zafrani, G. Sod-Moriah, D. Yeffet, A. Berliner, D. Amir, D. Marciano, S. Elias, S. Katalan, N. Ashkenazi and M. Madmon, et al., J. Med. Chem., 2019, 62, 5628-5637.

32 J. A. Erickson and J. I. McLoughlin, J. Org. Chem., 1995, 60, 1626-1631.

33 Y. Zafrani, D. Yeffet, G. Sod-Moriah, A. Berliner, D. Amir, D. Marciano, E. Gershonov and S. Saphier, J. Med. Chem., 2017, 60, 797-804.

34 C. D. Sessler, M. Rahm, S. Becker, J. M. Goldberg, F. Wang and S. J. Lippard, J. Am. Chem. Soc., 2017, 139, 9325-9332.

35 B. E. Smart, J. Fluorine Chem., 2001, 109, 3-11.

36 R. Pollice and P. Chen, J. Am. Chem. Soc., 2019, 141, 3489-3506.

37 I. T. Horváth and J. Rábai, Science, 1994, 266, 72-75.

38 J. G. Davis, K. P. Gierszal, P. Wang and D. Ben-Amotz, Nature, 2012, 491, 582-585.

39 K. P. Gierszal, J. G. Davis, M. D. Hands, D. S. Wilcox, L. V. Slipchenko and D. Ben-Amotz, J. Phys. Chem. Lett., 2011, 2, 2930-2933.

40 W. H. Lawton and E. A. Sylvestre, Technometrics, 1971, 13, 617-633.

41 P. Perera, M. Wyche, Y. Loethen and D. Ben-Amotz, J. Am. Chem. Soc., 2008, 130, 4576-4577.

42 J. R. Robalo, L. M. Streacker, D. Mendes de Oliveira, P. Imhof, D. Ben-Amotz and A. V. Verde, J. Am. Chem. Soc., 2019, 141, 15856-15868.

43 J. G. Davis, B. M. Rankin, K. P. Gierszal and D. Ben-Amotz, Nat. Chem., 2013, 5, 796.

44 H. W. Horn, W. C. Swope, J. W. Pitera, J. D. Madura, T. J. Dick, G. L. Hura and T. Head-Gordon, J. Chem. Phys, 2004, 120, 9665-9678.

45 J. Wang, R. M. Wolf, J. W. Caldwell, P. A. Kollman and D. A. Case, J. Comput. Chem., 2004, 25, 1157-1174.

46 J. R. Robalo, S. Huhmann, B. Koksch and A. Vila Verde, Chem, 2017, 3, 881-897.

47 P. Cieplak, W. D. Cornell, C. Bayly and P. A. Kollman, J. Comput. Chem., 1995, 16, 1357-1377.

48 M. J. Frisch, G. W. Trucks, H. B. Schlegel, G. E. Scuseria, M. A. Robb, J. R. Cheeseman, J. A. Montgomery, Jr., T. Vreven, K. N. Kudin, J. C. Burant, J. M. Millam, S. S. Iyengar, J. Tomasi, V. Barone, B. Mennucci, M. Cossi, G. Scalmani, N. Rega, G. A. Petersson, H. Nakatsuji, M. Hada, M. Ehara, K. Toyota, R. Fukuda, J. Hasegawa, M. Ishida, T. Nakajima, Y. Honda, O. Kitao, H. Nakai, M. Klene, X. Li, J. E. Knox, H. P. Hratchian, J. B. Cross, V. Bakken, C. Adamo, J. Jaramillo, R. Gomperts, R. E. Stratmann, O. Yazyev, A. J. Austin, R. Cammi, C. Pomelli, J. W. Ochterski, P. Y. Ayala, K. Morokuma, G. A. Voth, P. Salvador, J. J. Dannenberg, V. G. Zakrzewski, S. Dapprich, A. D. Daniels, M. C. Strain, O. Farkas, D. K. Malick, A. D. Rabuck, K. Raghavachari, J. B. Foresman, J. V. Ortiz, Q. Cui, A. G. Baboul, S. Clifford, J. Cioslowski, B. B. Stefanov, G. Liu, A. Liashenko, P. Piskorz, I. Komaromi, R. L. Martin, D. J. Fox, T. Keith, M. A. Al-Laham, C. Y. Peng, A. Nanayakkara, M. Challacombe, P. M. W. Gill, B. Johnson, W. Chen, M. W. Wong, C. Gonzalez and J. A. Pople, Gaussian 03, Revision E.01, 2004. 
49 J. Wang, W. Wang, P. A. Kollman and D. A. Case, J. Mol. Graphics Modell., 2006, 25, 247-260.

50 A. W. S. da Silva and W. F. Vranken, BMC Res. Notes, 2012, $5,1$.

51 D. A. Case, V. Babin, J. T. Berryman, R. M. Betz, Q. Cai, D. S. Cerutti, T. E. Cheatham III, T. A. Darden, R. E. Duke, H. Gohlke, A. W. Goetz, S. Gusarov, N. Homeyer, P. Janowski, J. Kaus, I. Kolossváry, A. Kovalenko, T. S. Lee, S. LeGrand, T. Luchko, R. Luo, B. Madej, K. M. Merz, F. Paesani, D. R. Roe, A. Roitberg, C. Sagui, R. SalomonFerrer, G. Seabra, C. L. Simmerling, W. Smith, J. Swails, R. C. Walker, J. Wang, R. M. Wolf, X. Wu and P. A. Kollman, AMBER 14, University of California, San Francisco, 2014.

52 H. Berendsen, D. van der Spoel and R. van Drunen, Comput. Phys. Commun., 1995, 91, 43-56.

53 E. Lindahl, B. Hess and D. van der Spoel, J. Mol. Model., 2001, 7, 306-317.

54 D. Van Der Spoel, E. Lindahl, B. Hess, G. Groenhof, A. E. Mark and H. J. C. Berendsen, J. Comput. Chem., 2005, 26, 1701-1718.

55 B. Hess, C. Kutzner, D. Van Der Spoel and E. Lindahl, J. Chem. Theory Comput., 2008, 4, 435-447.

56 S. Pronk, S. Páll, R. Schulz, P. Larsson, P. Bjelkmar, R. Apostolov, M. R. Shirts, J. C. Smith, P. M. Kasson, D. van der Spoel, B. Hess and E. Lindahl, Bioinformatics, 2013, 29, 845-854.

57 S. Páll, M. J. Abraham, C. Kutzner, B. Hess and E. Lindahl, International Conference on Exascale Applications and Software, 2014, pp. 3-27.

58 M. J. Abraham, T. Murtola, R. Schulz, S. Páll, J. C. Smith, B. Hess and E. Lindahl, SoftwareX, 2015, 1, 19-25.

59 U. Essmann, L. Perera, M. L. Berkowitz, T. Darden, H. Lee and L. G. Pedersen, J. Chem. Phys., 1995, 103, 8577-8593.

60 B. Hess, H. Bekker, H. J. C. Berendsen and J. G. E. M. Fraaije, J. Comput. Chem., 1997, 18, 1463-1472.

61 W. Humphrey, A. Dalke and K. Schulten, J. Mol. Graphics, 1996, 14, 33-38.

62 R. M. Parrish, L. A. Burns, D. G. A. Smith, A. C. Simmonett, A. E. DePrince, E. G. Hohenstein, U. Bozkaya, A. Y. Sokolov, R. Di Remigio, R. M. Richard, J. F. Gonthier, A. M. James, H. R. McAlexander, A. Kumar, M. Saitow, X. Wang, B. P. Pritchard, P. Verma, H. F. Schaefer, K. Patkowski, R. A. King, E. F. Valeev, F. A. Evangelista, J. M. Turney, T. D. Crawford and C. D. Sherrill, J. Chem. Theory Comput., 2017, 13, 3185-3197.

63 B. Jeziorski, R. Moszynski and K. Szalewicz, Chem. Rev., 1994, 94, 1887-1930.

64 E. G. Hohenstein and C. D. Sherrill, Wiley Interdiscip. Rev.: Comput. Mol. Sci., 2012, 2, 304-326.

65 R. M. Parrish, E. G. Hohenstein and C. D. Sherrill, J. Chem. Phys., 2013, 139, 174102.
66 E. G. Hohenstein, H. M. Jaeger, E. J. Carrell, G. S. Tschumper and C. D. Sherrill, J. Chem. Theory Comput., 2011, 7, 2842-2851.

67 E. G. Hohenstein and C. D. Sherrill, J. Chem. Phys., 2010, 133, 104107.

68 T. M. Parker, L. A. Burns, R. M. Parrish, A. G. Ryno and C. D. Sherrill, J. Chem. Phys., 2014, 140, 094106.

69 E. G. Hohenstein and C. D. Sherrill, J. Chem. Phys., 2010, 132, 184111.

70 E. G. Hohenstein and C. D. Sherrill, J. Chem. Phys., 2010, 133, 014101.

71 T. H. Dunning Jr, J. Chem. Phys., 1989, 90, 1007-1023.

72 R. A. Kendall, T. H. Dunning Jr and R. J. Harrison, J. Chem. Phys., 1992, 96, 6796-6806.

73 F. Weigend, A. Köhn and C. Hättig, J. Chem. Phys., 2002, 116, 3175-3183.

74 F. Weigend, Phys. Chem. Chem. Phys., 2002, 4, 4285-4291.

75 D. Marx and J. Hutter, Ab Initio Molecular Dynamics: Theory and Implementation, in Modern Methods and Algorithms of Quantum Chemistry, John von Neumann Institute for Computing, Jülich, 2000, vol. 3, pp. 329-477.

76 J. VandeVondele, M. Krack, F. Mohamed, M. Parrinello, T. Chassaing and J. Hutter, Comput. Phys. Commun., 2005, 167, 103-128.

77 J. Hutter, M. Iannuzzi, F. Schiffmann and J. VandeVondele, Wiley Interdiscip. Rev.: Comput. Mol. Sci., 2014, 4, 15-25.

78 G. Lippert, J. Hutter and M. Parrinello, Theor. Chem. Acc., 1999, 103, 124-140.

79 S. Grimme, J. Antony, S. Ehrlich and H. Krieg, J. Chem. Phys., 2010, 132, 154104.

80 M. Brehm and B. Kirchner, J. Chem. Inf. Model., 2011, 51, 2007-2023.

81 J. D. Hunter, Comput. Sci. Eng., 2007, 9, 90-95.

82 P. Orea, J. López-Lemus and J. Alejandre, J. Chem. Phys., 2005, 123, 114702.

83 M. González-Melchor, F. Bresme and J. Alejandre, J. Chem. Phys., 2005, 122, 104710.

84 F. G. J. Longford, J. W. Essex, C.-K. Skylaris and J. G. Frey, J. Chem. Phys., 2018, 148, 214704.

85 S. Roy, B. Biswas, N. Ghosh, P. C. Singh and J. A. Mondal, J. Phys. Chem. C, 2019, 123, 27012-27019.

86 H. C. Gottschalk, A. Poblotzki, M. A. Suhm, M. M. Al-Mogren, J. Antony, A. A. Auer, L. Baptista, D. M. Benoit, G. Bistoni, F. Bohle, R. Dahmani, D. Firaha, S. Grimme, A. Hansen, M. E. Harding, M. Hochlaf, C. Holzer, G. Jansen, W. Klopper, W. A. Kopp, L. C. Kröger, K. Leonhard, H. Mouhib, F. Neese, M. N. Pereira, I. S. Ulusoy, A. Wuttke and R. A. Mata, J. Chem. Phys., 2018, 148, 014301.

87 J. P. Wagner and P. R. Schreiner, Angew. Chem., Int. Ed., 2015, 54, 12274-12296.

88 R. A. Mata and M. A. Suhm, Angew. Chem., Int. Ed., 2017, 56, 11011-11018. 